\title{
A patient heterozygous for R92Q mutation with periodic fever and aphthous stomatitis, pharyngitis, and adenitis (PFAPA) syndrome-like phenotype
}

\author{
Ezgi Deniz Batu, Hafize Emine Sönmez, Yelda Bilginer, Seza Özen \\ Division of Rheumatology, Department of Pediatrics, Hacettepe University Faculty of Medicine, Ankara, Turkey. \\ E-mail: sezaozen@hacettepe.edu.tr \\ Received: 8th April 2017, Revised: 8th December 2017, Accepted: 24th December 2017
}

\begin{abstract}
SUMMARY: Batu ED, Sönmez HE, Bilginer Y, Özen S. A patient heterozygous for R92Q mutation with periodic fever and aphthous stomatitis, pharyngitis, and adenitis (PFAPA) syndrome-like phenotype. Turk J Pediatr 2018; 60: 726-728.

Tumor necrosis factor receptor associated periodic syndrome (TRAPS) is an autosomal dominant disease caused by mutations located on the type 1 tumor necrosis factor receptor (TNFRSF1A) gene. Here we present a 3-year-old boy heterozygous for $\mathrm{R} 92 \mathrm{Q}$ mutation in TNFRSF1A gene expressing a periodic fever, aphthous stomatitis, pharyngitis, and adenitis (PFAPA) syndrome-like phenotype. However, some of his symptoms such as myalgia and the long duration of fever attacks were not typical for PFAPA. He was treated with methylprednisolone during the attacks and also responded to colchicine. The family history revealed that his grandfather, mother, and uncle suffered from similar attacks, and interestingly all of them responded to tonsillectomy. PFAPA-like features have already been reported in patients with the R92Q mutation. However, this case is interesting with the response to colchicine treatment and response to tonsillectomy in his relatives.
\end{abstract}

Key words: periodic fever with aphthous stomatitis, pharyngitis, and adenitis, tumor necrosis factor receptor associated periodic syndrome, R92Q mutation.

Tumor necrosis factor receptor associated periodic syndrome (TRAPS) is an autosomal dominant disease caused by mutations located on the type 1 tumor necrosis factor receptor (TNFRSF1A) gene. ${ }^{1}$ Patients are characterized by long-lasting fever attacks, accompanied with rash, myalgia, abdominal pain, pericarditis, periorbital edema. ${ }^{2}$ More than 90 mutations have been described in TNFRSF1A, however the phenotype of the disease may be different in each individual (http://fmf.igh.cnrs.fr/infevers/). .3,4 Most of the mutations are structural mutations which have high penetrance and encode the extracellular cysteine-rich domain (CRD). However the R92Q variant is a missense and low-penetrance mutation without effect on the structure or function of the protein and has high frequency among Caucasian children with periodic fever. 5,6 This variant is associated with a milder phenotype characterized with short attack duration, high tendency to spontaneous resolution, lower prevalence of amyloidosis and less frequent family history of periodic fever. ${ }^{2,6}$ Here, we present a patient heterozygous for R92Q mutation in TNFRSF1A gene with periodic fever with aphthous stomatitis, pharyngitis, and adenitis (PFAPA) syndromelike phenotype.

\section{Case Report}

A 1-year-old boy presented with fever attacks, lasting 7-10 days with abdominal pain, arthralgia, aphthous stomatitis, pharyngitis, and myalgia. Initially, he was diagnosed with PFAPA, and received methylprednisolone (1 $\mathrm{mg} / \mathrm{kg} /$ day) during the attacks and his attacks improved with this treatment. His acute phase reactants were high. Although he was negative for mutations of the Mediterranean fever gene, colchicine was started at another center (0.5 $\mathrm{mg}$ /day) and the attacks improved with this 
therapy. Two years later, when he was 3 years old, he was admitted to our hospital. He had been suffering from a febrile attack lasting for 12 days and protracted myalgia. On physical examination, his temperature was $38.9^{\circ} \mathrm{C}$, he had aphthous stomatitis and bilateral cervical lymphadenopathy. His complete blood count showed hemoglobin was $12.3 \mathrm{~g} / \mathrm{dl}$ (11.5$14.5)$, white blood cell count $10800 / \mathrm{mm}^{3}$ (4000-12000), and platelet count was 326000/ $\mathrm{mm}^{3}$ (150000-450000). C-reactive protein and erythrocyte sedimentation rates were $4.6 \mathrm{mg} /$ dl (normal range, $0-0.8 \mathrm{mg} / \mathrm{dl}$ ), and $46 \mathrm{~mm}$ / hour (0-20), respectively. Muscle enzymes were normal [creatine kinase, $88(<145)$, lactate dehydrogenase, $232(<247)$, aspartate aminotransferase, $12(<52)]$.

Features such as myalgia and the long duration of fever attacks were considered atypical for PFAPA. A heterozygous p.Arg121Gln (c.312 $\mathrm{G}>\mathrm{A}$ ) (R92Q) mutation was detected in the TNFRSF1A gene. He responded to short term corticosteroid therapy ( $2 \mathrm{mg} / \mathrm{kg} /$ day; 4 days $)$. In the family history; he was the second child of consanguineous parents (first cousins) and his grandfather, mother, and uncle suffered from similar attacks, and interestingly the affected members had no attack after tonsillectomy. Parents, siblings (two sisters) and uncle of the patients were screened for mutations in TNFRSF1A gene. The patent's mother, uncle and one sister were heterozygous for p.Arg121Gln (c.312 G>A) (R92Q) mutation. However, the sister was healthy and had no symptoms related with periodic fever syndromes. This could be due to different penetrance of the mutation in different members of the family.

When the patient was admitted to the hospital, his parents gave informed consent approving genetic analyzes and anonymous data use for academic purpose.

\section{Discussion}

Patients heterozygous for R92Q mutation display various phenotypes ranging from TRAPS to PFAPA-like symptoms. Pelagatti et al. ${ }^{6}$ compared the clinical and laboratory features of TRAPS patients (including 11 patients with structural mutations and 20 patients with R92Q) with PFAPA patients and found that phenotypes of TRAPS patients with R92Q mutation might resemble PFAPA; however, PFAPA patients had significantly shorter attack period, more frequent attacks per year, earlier age of disease onset, and higher rate of periodicity than PFAPA-like TRAPS patients. Patients with structural mutations had frequently more severe symptoms such as abdominal pain, skin rash, and myalgia. They also reported that a significant portion of patients with R92Q mutation experienced spontaneous resolution (25\%) or amelioration of fever attacks (56\%) like PPAFA patients. ${ }^{6}$ Our patient had an early disease onset like PFAPA; however the duration of fever attacks was long and the fever was usually accompanied with myalgia. Family history of TRAPS is more frequent in patients who carried structural mutations than patients with R92Q mutation. ${ }^{2,6}$ However, our patient had three affected family members. In previous studies, it was shown that patients with R92Q mutation had good response to short term steroid (ondemand therapy).2,6 Interestingly, our patient improved with colchicine treatment. However, spontaneous remission was also reported in patients with R92Q heterozygous mutation. One more case with R92Q mutation had been reported from our center with similar features of PFAPA. ${ }^{7}$

The interesting features of our case were improvement with colchicine treatment and response to tonsillectomy in his relatives. Patients with R92Q mutation of TNFRSF1A may display milder phenotype and resemble PFAPA.

\section{REFERENCES}

1. McDermott MF, Aksentijevich I, Galon J, et al. Germline mutations in the extracellular domains of the $55 \mathrm{kDa}$ TNF receptor, TNFR1, define a family of dominantly inherited autoinflammatory syndromes. Cell 1999; 97: 133-144.

2. Lachmann HJ, Papa R, Gerhold K, et al. Paediatric Rheumatology International Trials Organisation (PRINTO), the EUROTRAPS and the Eurofever Project. The phenotype of TNF receptor-associated autoinflammatory syndrome (TRAPS) at presentation: A series of 158 cases from the Eurofever/EUROTRAPS international registry. Ann Rheum Dis 2014; 73: 21602167.

3. Milhavet F, Cuisset L, Hoffman HM, et al. The infevers autoinflammatory mutation online registry: Update with new genes and functions. Hum Mutat 2008; 29: 803-808. 
4. Touitou I, Lesage S, McDermott M, et al. Infevers: An evolving mutation database for auto-inflammatory syndromes. Hum Mutat 2004; 24: 194-198.

5. Lainka E, Neudorf $U$, Lohse $P$, et al. Incidence of TNFRSF1A mutations in German children: Epidemiological, clinical and genetic characteristics. Rheumatology (Oxford) 2009; 48: 987-991.
6. Pelagatti MA, Meini A, Caorsi R, et al. Long-term clinical profile of children with the low-penetrance R92Q mutation of the TNFRSF1A gene. Arthritis Rheum 2011; 63: 1141-1150.

7. Celebi-Tayfur A, Bilginer Y, Finetti M, Gattorno M, Ozen S. Different presentations in patients with tumor necrosis factor receptor-associated periodic syndrome mutations: Report of two cases. Turk J Pediatr 2013; 55: 78-81. 